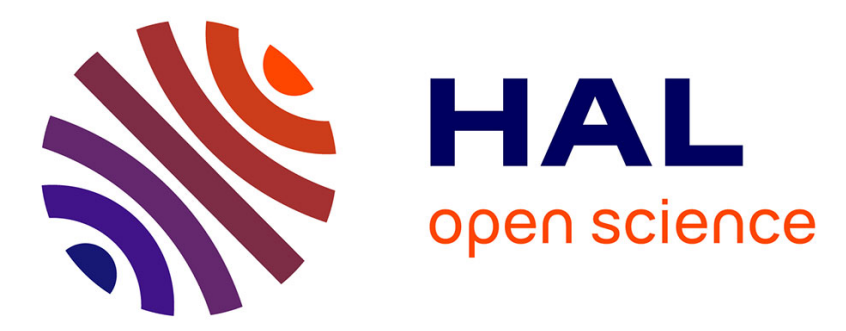

\title{
Polariton topological transition effects on radiative heat transfer
}

Cheng-Long Zhou, Xiao-Hu Wu, Yong Zhang, Hing-Liang Yi, Mauro Antezza

\section{To cite this version:}

Cheng-Long Zhou, Xiao-Hu Wu, Yong Zhang, Hing-Liang Yi, Mauro Antezza. Polariton topological transition effects on radiative heat transfer. Physical Review B, 2021, 103, pp.155404. 10.1103/PhysRevB.103.155404 . hal-03191119

\section{HAL Id: hal-03191119 https://hal.science/hal-03191119}

Submitted on 25 May 2021

HAL is a multi-disciplinary open access archive for the deposit and dissemination of scientific research documents, whether they are published or not. The documents may come from teaching and research institutions in France or abroad, or from public or private research centers.
L'archive ouverte pluridisciplinaire HAL, est destinée au dépôt et à la diffusion de documents scientifiques de niveau recherche, publiés ou non, émanant des établissements d'enseignement et de recherche français ou étrangers, des laboratoires publics ou privés. 


\title{
Polariton topological transition effects on radiative heat transfer
}

\author{
Cheng-Long Zhou, ${ }^{1,2}$ Xiao-Hu Wu $\odot,{ }^{3}$ Yong Zhang, ${ }^{1,2}$ Hong-Liang Yi $\odot,{ }^{1,2, *}$ and Mauro Antezza $\odot^{4,5, \dagger}$ \\ ${ }^{1}$ School of Energy Science and Engineering, Harbin Institute of Technology, Harbin 150001, People's Republic of China \\ ${ }^{2}$ Key Laboratory of Aerospace Thermophysics, Ministry of Industry and Information Technology, Harbin 150001, People's Republic of China \\ ${ }^{3}$ Shandong Institute of Advanced Technology, Jinan 250100, Shandong, China \\ ${ }^{4}$ Laboratoire Charles Coulomb (L2C), UMR 5221 CNRS-Université de Montpellier, F-34095 Montpellier, France \\ ${ }^{5}$ Institut Universitaire de France, 1 rue Descartes, F-75231 Paris, France
}

(Received 26 October 2020; revised 25 February 2021; accepted 19 March 2021; published 6 April 2021)

\begin{abstract}
Twisted two-dimensional bilayer anisotropy materials exhibit many exotic physical phenomena. Manipulating the "twist angle" between the two layers enables the hybridization phenomenon of polaritons, resulting in fine control of the dispersion engineering of the polaritons in these structures. Here, combined with the hybridization phenomenon of anisotropy polaritons, we study theoretically the near-field radiative heat transfer (NFRHT) between two twisted hyperbolic systems. These two twisted hyperbolic systems are mirror images of each other. Each twisted hyperbolic system is composed of two graphene gratings, where there is an angle $\varphi$ between these two graphene gratings. By analyzing the photonic transmission coefficient as well as the plasmon dispersion relation of the twisted hyperbolic system, we prove the enhancement effect of the topological transitions of the surface state at a special angle [from open (hyperbolic) to closed (elliptical) contours] on radiative heat transfer. Meanwhile the role of the thickness of dielectric spacer and vacuum gap on the manipulating the topological transitions of the surface state and the NFRHT are also discussed. We predict the hysteresis effect of topological transitions at a larger vacuum gap, and demonstrate that as the thickness of the dielectric spacer increases, the transition from the enhancement effect of heat transfer caused by the twisted hyperbolic system to a suppression.
\end{abstract}

DOI: 10.1103/PhysRevB.103.155404

\section{INTRODUCTION}

Since the pioneering work of Polder and Hove [1], when two objects are placed at a distance comparable to or shorter than the characterized thermal radiation wavelength, i.e., in the near-field regime, the forward and backward evanescent waves can couple with each other and open paths for photons to tunnel through [2-4]. This phenomenon is called photon tunneling and there are more tunneling photons than propagating photons, resulting in a radiative heat transfer that can be orders of magnitude higher than the blackbody limit by Planck's law. The huge radiative heat flux in the near field opens the door to various applications like thermophotovoltaic devices [5-8], thermal rectification [9,10], information processing [11], and noncontact refrigeration [12]. Among these, the coupling of surface polaritons plays a decisive role in huge heat transfer enhancement [4]. In particular, the nearfield radiative heat transfer (NFRHT) can be far ahead of the blackbody limit, either theoretically or experimentally, via the resonant coupling of surface phonon polaritons (SPhPs) [13] or surface plasmon polaritons (SPPs) [14]. Moreover, the development in fabrication of metamaterials results in extensive studies of the coupling of surface polaritons for NFRHT between metamaterials in theory, such as hyperbolic polaritons [15], magnetoplasmon polaritons [16], ellipse polaritons [17], nonreciprocal polaritons [18], and nonreciprocal hyperbolic

\footnotetext{
*Corresponding author: yihongliang@ @it.edu.cn

${ }^{\dagger}$ Corresponding author: mauro.antezza@umontpellier.fr
}

polaritons [19]. Various types of surface polaritons have been extensively studied for their ability to dominate the photon tunneling and greatly modulate the near-field heat transfer.

Moreover, since the hybridization effect of polaritons can greatly change the optical performance of the system, continuous efforts have been devoted to exploring the influence of hybridization of polaritons on heat transfer. For example, surface plasmons in graphene can couple with hyperbolic phonon polaritons in an $\mathrm{hBN}$ film to form hybrid polaritons that can assist photon tunneling [20]. The hybridization effect between surface plasmon polaritons of graphene and semiconductor can well alleviate the suppression effect of the decoupling characteristics of the asymmetry system on the near-field radiation heat transfer [21]. At a strong magnetic field, the coupling of magnetoplasmon polaritons from graphene to surface phonon polaritons from $\mathrm{SiO}_{2}$ can reach a high thermal magnetoresistance to impede near-field radiative heat transfer [22].

These strategies have largely explored the influence of the hybridization effect of polaritons on near-field thermal radiation [20-27]. Nonetheless, up to now, the influence of the hybridization of anisotropic polaritons on the near-field radiation remains elusive. Recently, since the pioneering work of $\mathrm{Hu}$ et al., twisted bilayer two-dimensional (2D) materials, in which one layer is rotated with respect to the other, enable the hybridization phenomenon of polaritons, resulting in fine control of the dispersion engineering of the polaritons in these structures [28,29]. Inspired by this concept, we explore heat transfer based on the twisted hyperbolic system, in which an interesting mechanism of hybridization of anisotropic 
polaritons may be introduced for NFRHT. The twisted hyperbolic system is composed of stacked uniaxial supporting hyperbolic polaritons. The uniaxial hyperbolic metasurfaces can be demonstrated in several material platforms, from Waals materials [30] to black phosphorus [31,32] and other 2D materials. Moreover, the surface characteristics of objects can be anisotropic when they are patterned into metasurfaces or gratings. Especially, by patterning a single layer of graphene sheet into ribbons, the closed circular dispersion of graphene plasmons is opened to become hyperbolic, leading to a strong NFRHT [33]. Since the strong NFRHT of graphene nanoribbons, continuous efforts have been devoted to exploit the heat transfer phenomenon of graphene nanoribbons. For example, the hyperbolic characteristics of a monolayer graphene grating can be adjusted by magnetic field [34] and current [19] to modulate its near-field radiation heat transfer. Since graphene nanoribbons that can provide robust hyperbolic polaritons in a board frequency band, we consider densely packed graphene nanoribbons as the platform of choice.

In this work, we theoretically investigate the influence of hybridization of anisotropic hyperbolic polaritons on NFRHT between two bilayer graphene nanoribbons. Through tailored interlayer coupling in twisted bilayer, the hyperbolic polaritons undergoes topological transitions at transitional angles, that is, the polaritons change markedly in nature from hyperbolic (open) to elliptical (closed). We investigate the effects of the topological transitions of hyperbolic polaritons on the radiative heat flux and, in particular, what happens to the hyperbolic modes of the twisted structure. In addition, the effects of the thickness of dielectric spacer and vacuum gap on the NFRHT are discussed at the end.

\section{THEORETICAL ASPECTS}

Let us consider a system composed of two twisted hyperbolic systems brought into close proximity with a vacuum gap size of $d_{0}$ as sketched in Fig. 1(a). We can form a twisted hyperbolic system by coupling two identical graphene nanoribbons, as sketched in Fig. 1(a), separated by thickness $d_{s}$ of the dielectric spacer. Each graphene strip has width $W$ and an air gap $G$ separates neighboring strips. We define the graphene strips of the twisted hyperbolic system above the vacuum gap as $G_{1}$ and $G_{2}$ from top to bottom, and the graphene strips of the twisted hyperbolic system below the vacuum gap as $G_{3}$ and $G_{4}$ from top to bottom. The upper structure is the emitter $\left(G_{1}\right.$ and $\left.G_{2}\right)$ with a higher temperature $T_{1}$ and the bottom structure $\left(G_{3}\right.$ and $\left.G_{4}\right)$ is the receiver with a lower temperature $T_{2}$. The temperatures are set to be $T_{1}=310 \mathrm{~K}$ and $T_{2}=300 \mathrm{~K}$, respectively. Here, $\alpha$ and $\beta$ are defined perpendicular to periodical directions of the first layer and the second layer in the one bilayer, respectively. Here, the first layer represents the graphene strips close to the vacuum gap, and the second layer represents the strips away from the vacuum gap. When the second layer in one bilayer system can be rotated with respect to the $y$ axis by an angle of $\varphi$ from $0^{\circ}$ to $90^{\circ}$, as shown in Figs. 1(b) and 1(c), an interesting effects of twisted periodicities is achieved. For simplicity, we assume that the emitter and receiver are mirror images of each other and the background materials to be vacuum. On the other word, we rotate simultaneously an angle of $\varphi$ between

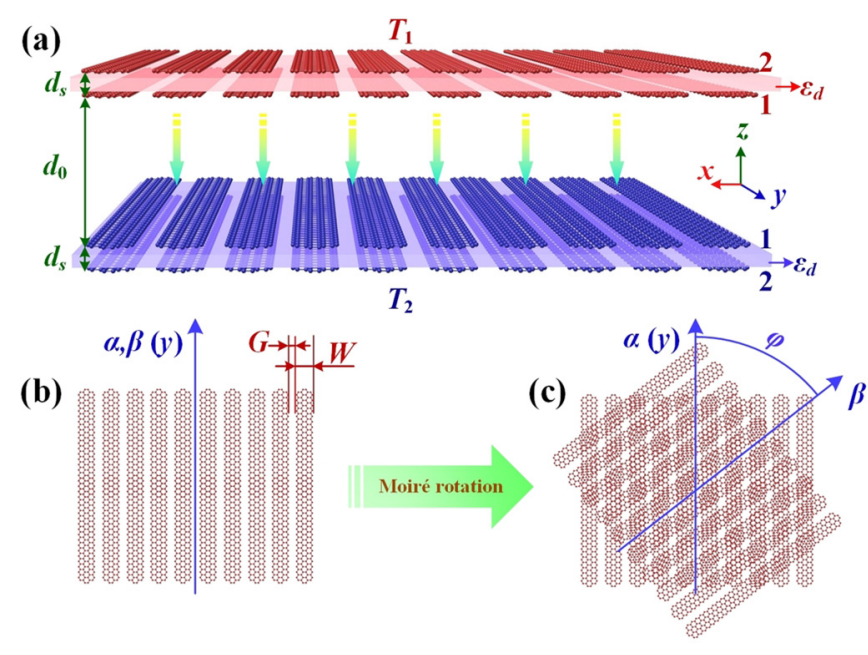

FIG. 1. (a) Schematic of near-field heat transfer between two twisted hyperbolic systems. The twisted hyperbolic system is composed of two identical graphene nanoribbons, whose width and air gap are $W$ and $G$, respectively. The structure above the vacuum gap $\left(G_{1}\right.$ and $\left.G_{2}\right)$ is the emitter with a higher temperature $T_{1}(310 \mathrm{~K})$ and the structure below the vacuum gap $\left(G_{3}\right.$ and $\left.G_{4}\right)$ is the receiver with a lower temperature $T_{2}(300 \mathrm{~K})$. (b) Top view of the crystalline structure without rotation. (c) Top view of the twisted hyperbolic system at a rotation angle $\varphi$ of with respect to $y$ axis.

$G_{1}$ and $G_{2}$ and an angle of $\varphi$ between $G_{3}$ and $G_{4}$. Here, we strictly compliance the emitter and receiver are mirror images of each other, that is, keeping $G_{2}$ parallel to $G_{3}$ and $G_{1}$ parallel to $G_{4}$. The nonmirrored system and dielectric backgrounds can be easily captured by generalizing the formulation in this work. In practical applications, we note that the twisted bilayer graphene grating can be implemented experimentally in the following procedure: the bottom graphene grating can be fabricated by electron beam lithography or chemical etching on the basement [35]. Then, the dielectric spacer between two graphene gratings can be prepared through chemical vapor deposition [29]. Through mechanical exfoliation, the top graphene sheet is transferred onto the dielectric spacer [36]. Last, the top graphene grating also can be fabricated by electron beam lithography or chemical etching on the dielectric spacer. By separately controlling the direction of the electron beam or the direction of chemical etching during the manufacturing process of the top and bottom gratings, the twist angle in the bilayer twisted grating system can be realized.

The optical conductivity of the graphene nanoribbon array of the present work is derived using a well-known effective medium theory (EMT) [37-39] that holds when the unitcell period $P=W+G$ is much smaller than the operating wavelength, i.e., $P \ll \lambda_{0}$. Meanwhile, in Ref. [33], EMT is valid only when the vacuum gap is larger than the period of the ribbons. When the gap distance is several times greater than the period, EMT can predict the real heat flux well, as quantitatively shown in Ref. [33]. According to Ref. [33], when the vacuum gap $d_{0}$ is $50 \mathrm{~nm}$ and the period $P$ is $10 \mathrm{~nm}$, the error by EMT is less than $6.69 \%$ compared with rigorous coupled-wave analysis (RCWA). As the gap distance increases, the accuracy of EMT can be further improved [33]. However, for the unit-cell period of $10 \mathrm{~nm}$, EMT may fail to 
predict the NFRHT when the gap distance is less than $50 \mathrm{~nm}$, and the results predicted by EMT will far exceed those calculated by RCWA [33]. Therefore, for the unit-cell period of $10 \mathrm{~nm}$, the vacuum gap greater than $50 \mathrm{~nm}$ is the limit of validity for this approximation [33]. In this paper we strictly ensure that the vacuum gap is greater than $50 \mathrm{~nm}$. The EMT is based on modeling the strip near-field coupling as an effective capacitance [33],

$$
\sigma_{\text {eff }}=\left[\begin{array}{cc}
\sigma_{x x} & 0 \\
0 & \sigma_{y y}
\end{array}\right]=\left[\begin{array}{cc}
\sigma_{x x}=\frac{P \sigma_{C} \sigma_{G}}{\left(W \sigma_{C}+G \sigma_{G}\right)} & 0 \\
0 & \frac{W}{P} \sigma_{G}
\end{array}\right],
$$

where $\sigma_{C}=-i \omega \varepsilon_{0} P /(\pi \ln \{\csc [0.5 \pi(P-W) / P]\})$ is the effective strip conductivity taking into account near-field coupling and nonlocality. In the hyperbolic regime, the metasurface acts as a metal for one transverse field polarization $\left(\operatorname{Im}\left[\sigma_{y y}\right]>0\right)$ and as a dielectric for the other one $\left(\operatorname{Im}\left[\sigma_{x x}\right]<0\right) . \omega$ is the frequency, and $\varepsilon_{0}$ is the vacuum permittivity. $\sigma_{\mathrm{G}}$ is the optical conductivity of the graphene, and it is given by the wellknown random phase approximation. Following Ref. [33], the conductivity can be written as a sum of intraband (Drude) and interband contributions, i.e., $\sigma_{G}=\sigma_{D}+\sigma_{I}$, respectively,

$$
\begin{aligned}
\sigma_{D} & =\frac{i}{\omega+i / \tau} \frac{2 e^{2} k_{B} T}{\pi \hbar^{2}} \ln \left(2 \cosh \frac{\mu}{2 k_{B} T}\right) \\
\sigma_{I} & =\frac{e^{2}}{4 \hbar}\left[G\left(\frac{\hbar \omega}{2}\right)+i \frac{4 \hbar \omega}{\pi} \int_{0}^{\infty} \frac{G(\xi)-G(\hbar \omega / 2)}{(\hbar \omega)^{2}-4 \xi^{2}} d \xi\right]
\end{aligned}
$$

where $G(\xi)=\sinh \left(\xi / k_{B} T\right) /\left[\cosh \left(E_{f} / k_{B} T\right)+\cosh \left(\xi / k_{B} T\right)\right]$. The conductivity depends explicitly on the temperature $T$ and the chemical potential $\mu$. The relaxation time $\tau$ is fixed at $10^{-13} \mathrm{~s}[33]$.

The radiative heat flux between two twisted hyperbolic systems can be calculated based on the fluctuation-dissipation theory [19],

$$
\begin{aligned}
q= & \int_{0}^{\infty} q_{\omega}(\omega) d \omega=\int_{0}^{\infty}\left[\Theta\left(\omega, T_{1}\right)-\Theta\left(\omega, T_{2}\right)\right] d \omega \\
& \times \int_{-\infty}^{\infty} \int_{-\infty}^{\infty} \frac{\xi\left(\omega, k_{x}, k_{y}\right)}{8 \pi^{3}} d k_{x} d k_{y},
\end{aligned}
$$

where $\Theta(\omega, T)=\hbar \omega /\left[\exp \left(\hbar \omega / k_{B} T\right)-1\right]$ is the mean energy of a Planck oscillator at angular frequency $\omega . \xi\left(\omega, k_{x}, k_{y}\right)$ is the photonic transmission coefficient (PTC) that describes the probability of photons excited by thermal energy, which can be written as

$$
\begin{aligned}
& \xi\left(\omega, k_{x}, k_{y}\right) \\
& =\left\{\begin{array}{l}
\operatorname{Tr}\left[\left(\mathbf{I}-\mathbf{R}_{2}^{*} \mathbf{R}_{2}-\mathbf{T}_{2}^{*} \mathbf{T}_{2}\right) \mathbf{D}\left(\mathbf{I}-\mathbf{R}_{1} \mathbf{R}_{1}^{*}-\mathbf{T}_{1}^{*} \mathbf{T}_{1}\right) \mathbf{D}^{*}\right], k<k_{0} \\
\operatorname{Tr}\left[\left(\mathbf{R}_{2}^{*}-\mathbf{R}_{2}\right) \mathbf{D}\left(\mathbf{R}_{1}-\mathbf{R}_{1}^{*}\right) \mathbf{D}^{*}\right] e^{-2\left|k_{z}\right| d}, k>k_{0}
\end{array}\right.
\end{aligned}
$$

for propagating $\left(k<k_{0}\right)$ and evanescent $\left(k>k_{0}\right)$ waves where $k=\sqrt{k_{x}^{2}+k_{y}^{2}}$ is the surface parallel wave vector and $k_{0}=\omega / c$ is the wave vector in vacuum. $k_{z}=\sqrt{k_{0}^{2}-k^{2}}$ is the tangential wave vector along the $z$ direction in vacuum and ${ }^{*}$ signifies the complex conjugate. The $2 \times 2$ matrix $\mathbf{D}$ is defined as $\mathbf{D}=\left(\mathbf{I}-\mathbf{R}_{1} \mathbf{R}_{2} e^{2 i k_{z} d}\right)$ which describes the
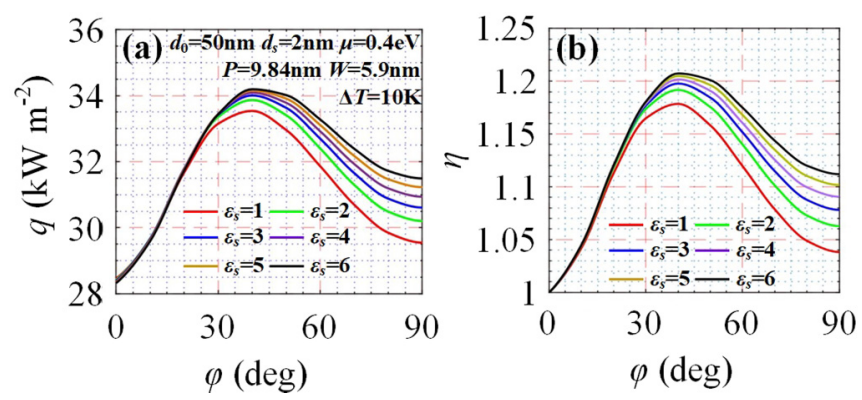

FIG. 2. (a) Radiative heat flux $q$ as a function of rotation angle $\varphi$ at different dielectric permittivity of spacer. (b) Heat transfer coefficient ratio $\eta$ between hyperbolic system and nonrotating system as a function of rotation angle $\varphi$ at different dielectric permittivity of spacer. The parameters are $d_{0}=50 \mathrm{~nm}, d_{s}=1 \mathrm{~nm}, T_{1}=310 \mathrm{~K}$, $T_{2}=300 \mathrm{~K}, \mu=0.4 \mathrm{eV}, W=5.9 \mathrm{~nm}$, and $P=9.84 \mathrm{~nm}$.

usual Fabry-Perot-like denominator resulting from the multiple scattering between the two interfaces of receiver and emitter. The reflection matrix $\mathbf{R}$ is a $2 \times 2$ matrix in the polarization representation; the calculation process of $\mathbf{R}$ can be found in Appendix A.

\section{HYPERBOLIC HYBRIDIZATION GUIDED HEAT TRANSFER BETWEEN TWO BILAYER GRAPHENE GRATING SYSTEMS}

We first discuss the radiative heat flux (RHF) between the two bilayer graphene grating systems as a function of the rotation angle $\varphi$ by fixing the vacuum gap $d_{0}$ to the value of $50 \mathrm{~nm}$ as shown in Fig 2. We fix the thickness of dielectric spacer to $d_{s}=1 \mathrm{~nm}$. Here, it is worth noting that the thickness of the dielectric gasket cannot be reduced indefinitely. If the spacing between two graphene layers is within an atomic lattice $(0.35 \mathrm{~nm})$, quantum coupling will occur between the two graphene layers [40]. Their conductivity description based on the random phase approximation no longer is applicable and should be modified accordingly to accommodate nonlocal and other tunneling effects [40]. However, for hypothetical twisted atomic bilayers, if their interlayer distance is relatively large, namely, thickness of the dielectric spacer is larger than 0.35 $\mathrm{nm}$, the interlayer quantum coupling would disappear [40]. Thus, in the following sections, we always keep the thickness of the dielectric spacer greater than $0.35 \mathrm{~nm}$. The chemical potential $\mu$ is fixed at $0.4 \mathrm{eV}$. Here, the chemical potential can be set by nitric acid chemical doping [41] or an external bias voltage [42]. In the nature, the width of graphene stripe should be a multiple of the lattice constant $a$ of graphene $(a=0.246 \mathrm{~nm})$. We consider that the width $W$ is $24 a(5.9 \mathrm{~nm})$ and the unit-cell period $P$ is $40 a(9.84 \mathrm{~nm})$, which can truly describe graphene grating. Here, besides, we also illustrate the impact of the dielectric permittivity $\varepsilon_{s}$ of the spacer with respect to the rotation angle $\varphi$ on the results.

We can see in the Fig. 2(a) that the presence of the rotation angle significantly modifies the RHF between the two bilayer graphene grating systems. In most of the cases, the RHF is higher than the case without rotation angle, which means that this torsion can enhances effectively the heat transfer. It can be seen more intuitively through the heat transfer coefficient 

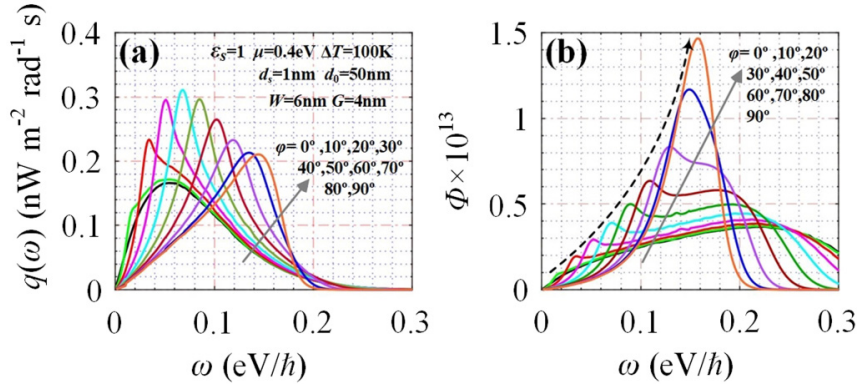

FIG. 3. (a) Spectral RHF as a function of the frequency for a vacuum gap $d_{0}=50 \mathrm{~nm}$ and a chemical potential $\mu=0.4 \mathrm{eV}$. The different lines correspond to different rotation angles. (b) Contour plots of energy transfer function $\Phi(\omega)$, as a function of frequency with different rotation angles.

ratio in Fig. 2(b) that the twisted system can enhance the radiative heat transfer of the system, and the enhancement would increase as the dielectric permittivity of spacer increases. However, as shown in Fig. 2(a), we find that the RHF exhibits a nonmonotonic dependency versus the rotation angle, especially for a lower dielectric permittivity of spacer. For the dielectric permittivity of a spacer of 1 , when the rotation angle reaches $40^{\circ}$, the maximum of the RHF increases from $28.3 \mathrm{~kW} \mathrm{~m}^{-2}$ for $\phi=0^{\circ}$ to around $33.5 \mathrm{~kW} \mathrm{~m}^{-2}$ for $\phi=40^{\circ}$ $(\eta=1.18)$, and decreases rapidly as the rotation angle further increases. In addition, it can be seen that although the dielectric permittivity of the spacer between two graphene gratings could tune the RHF of a metastructure, the dependency of RHF versus the rotation angle under different dielectric permittivity has been constant. In order to conveniently reveal the physical mechanism of a twisted hyperbolic system in NFRHT, we fixed the dielectric permittivity of the spacer to 1 in the subsequent analysis.

To get further insight into the role of the twisted hyperbolic system, we show in Fig. 3(a) the spectral RHF for a gap $d_{0}=50 \mathrm{~nm}$, the dielectric permittivity of the spacer is 1 , and different rotation angles. This spectral RHF is defined as the RHF per unit of frequency. It can be seen that a new peak appears as the twisted hyperbolic system emerges $\left(\varphi>0^{\circ}\right)$. In addition, when the rotation angle increases to $20^{\circ}$, the new peak begin to dominate the spectral RHF, that is, the new peak becomes the maximum of the spectral RHF. The new peak of the spectral RHF is blueshifted upon increasing the rotation angle from $0.018 \mathrm{eV} / \hbar$ for $\varphi=10^{\circ}$ up to around $0.145 \mathrm{eV} / \hbar$ for $\varphi=90^{\circ}$. Notice also that the maximum of the spectral RHF also increases drastically with the rotation angle, reaching a maximum of $0.31 \mathrm{nW} \mathrm{m}^{-2} \mathrm{rad}^{-1} \mathrm{~s}$ at $\varphi=40^{\circ}$. As the rotation angle further increases, the maximum of the spectral RHF gradually decreases to the value of $0.21 \mathrm{nW} \mathrm{m}^{-2} \mathrm{rad}^{-1} \mathrm{~s}$ at $\varphi=90^{\circ}$.

In order to visualize the NFRHT of the rotation angle, in Fig. 3(b), we calculate the energy transfer function $\Phi(\omega)$, given by $\Phi(\omega)=q_{\omega} /\left[\Theta\left(\omega, T_{1}\right)-\Theta\left(\omega, T_{2}\right)\right]$. As the rotation angle increases, the peak of energy transfer function generated by the twisted hyperbolic system increases drastically, reaching a maximum of $1.5 \times 10^{13}$. This also explains that the spectral RHF in Fig. 3(b) increases as the rotation angle increases at the lower rotation angle range. However, the peak of energy transfer function generated by higher rotation angle is excited only by higher photonic energy, which corresponds to the blueshifted peak of spectral RHF shown in Fig. 3(a). Unfortunately, the contribution of spectral RHF with higher frequency (higher photonic energy) to RHF is negligible, due to the exponentially decaying mean energy of a Planck oscillator $\Theta(\omega, T)$ at room temperature. Therefore, in Fig. 3(a), when the rotation angle is $90^{\circ}$, it is difficult to observe the enhanced spectral RHF excited by the ultrahigh energy transfer coefficient, which is in agreement with the declining trend of the RHF at the larger rotation angle range in Fig. 2.

\section{UNDERLYING PHYSICS OF THE HYPERBOLIC HYBRIDIZATION}

It is well known that the photonic transmission coefficient (PTC) and dispersion relation can visually reveal the physical mechanism of the effect of surface state on NFRHT. We thus employed these two physical quantities to explore the underlying physical mechanisms for this twisted hyperbolic system to the NFRHT. For a frequency of $0.15 \mathrm{eV} / \hbar$, the lower panels of Figs. 4(b)-4(f) show the distributions of the photonic transmission coefficient and the dispersion relation with rotation angle $\varphi=0^{\circ}, 30^{\circ}, 60^{\circ}, 80^{\circ}$, and $90^{\circ}$, respectively. As we all know, different from conventional circles, the surface state of anisotropic SPPs supported by graphite gratings graphs as a hyperbola, where the dominant contribution to the optical response of graphene comes from the scattering of free electrons. As shown in Fig. 4(b), the regime featured with PTC for the bilayer graphene grating system is axisymmetric hyperbolic lines. The dispersion relation of effective graphene gratings can well reflect this phenomenon, and it is given by Eq. (A11). Moreover, as the two bodies are brought into proximity, the evanescent field of HSPPs associated with two graphene gratings can interact with each other, leading to the two dispersion curves at the higher wave-vector region and the smaller wave-vector region. However, due to the existence of the attenuation length $\left[\delta_{z}=1 / \operatorname{Im}\left(k_{z}\right)\right]$ [18], the surface state dominated by dispersion at the higher wave-vector region is easily filtered by the vacuum gap, and cannot make a corresponding contribution to the NFRHT. Therefore, in the subsequent analysis, we only show the dispersion relationship at the smaller wave-vector region, unless otherwise stated.

As shown in Figs. 4(b)-4(f), it can be clearly seen that when the second individual graphene grating of a bilayer system emerges with mechanical rotation, the bright branch of the polaritons changes markedly-from hyperbolic (open) to elliptical (closed). This photonic topological transition is governed by the coupling of in-plane hyperbolic SPPs that are individually supported by each layer of the bilayer system [28]. Before the rotation angle at which the topological transition arises, as the rotation angle increases, gradually the hyperbolic bright branch would be flattened. After a topological transition occurs on the surface state (i.e., the bright band closes), the surface state gradually change from quasielliptical plasmon to quasi-isotropic plasmon with the rotation angle increase. Moreover, as its surface state changes from open to closed, the topological transition yields diffraction less, and low-loss directional SPPs become canalized, which in turn produces a more intense surface state, i.e., a brighter and 


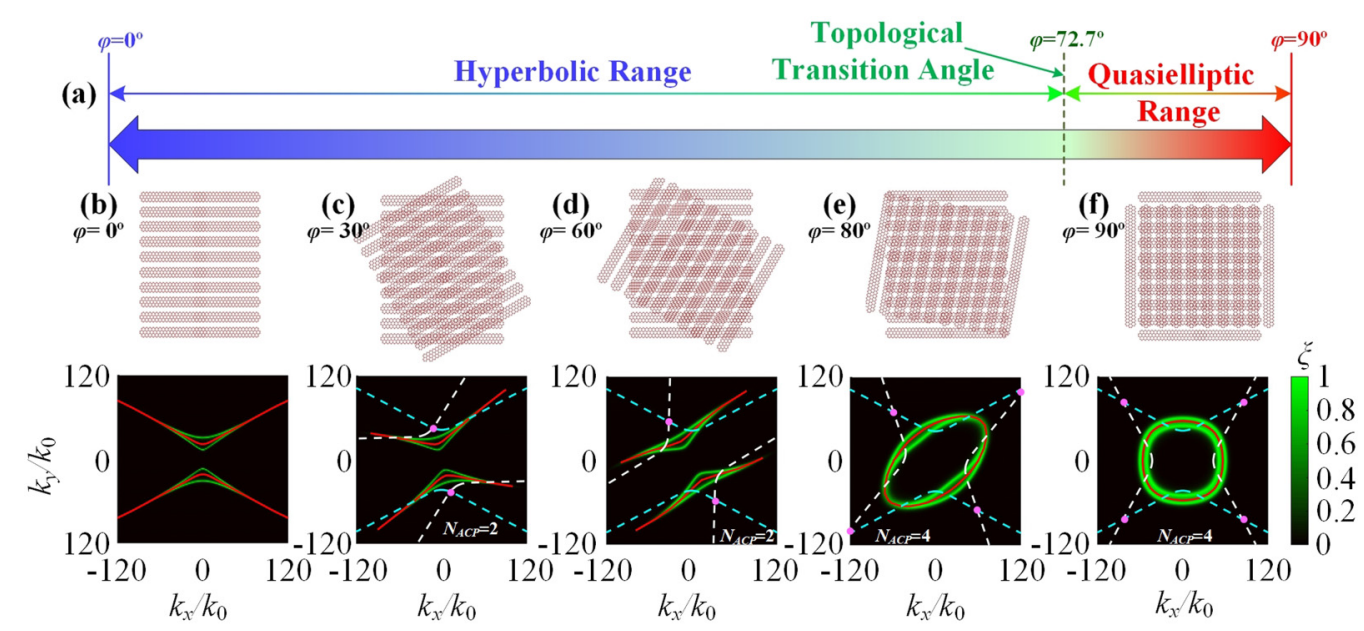

FIG. 4. Rotation-induced topological transition of plasmon polaritons. (a) Topological nature of the plasmon polaritons dispersion with respect to the rotation angle at a frequency of $0.15 \mathrm{eV} / \hbar$. (b)-(f) The top row shows the formed twisted fringe patterns of twisted bilayer hyperbolic system. The bottom row is the numerically simulated photonic transmission coefficient in wave-vector space. The blue dashed lines and the white dashed lines are the dispersion lines of the top grating (no rotation) and bottom grating (with rotation) of twisted bilayer system. The red solid lines are the dispersion after hybridization of twisted hyperbolic system.

stronger bright branch, as shown in Figs. 4(b)-4(f). These results contribute to a further confirmation and explanation of the increase of the energy transfer function in Fig. 3(b). Furthermore, it should be emphasized that the topological transition of the surface state is completely different from the previous work about controlling NFRHT based on the mechanical rotating between the emitter and the receiver. The topological transition of the surface state is a nonlinear change caused by the twisted hybridization based on the twisted hyperbolic system. The previous work about the mechanical rotating between the emitter and the receiver is just a simple superposition of surface state, and the corresponding understanding is linear change [43-47].

In addition, to explore thoroughly these effects, Figs. 4(b)4(f) show the isofrequency dispersion curves for the twisted hyperbolic system considering different rotation angles. The red solid lines show the calculated dispersion bands of the twisted hyperbolic system. The blue and white dashed lines correspond to the dispersion curves of the isolated top (no rotation) and bottom (with rotation) graphene gratings. This red solid line nicely locates at the bright branches, which unambiguously demonstrates that topological hybridization of HSPPs dominate the NFRHT in our system. In addition, we can predict this transition of the bright branch via the number of crossing points $N_{A C P}$ for the dispersion lines of each isolated graphene grating in bilayer system, analogous to a Lifshitz transition in electronics $[28,29]$. The bright branch of the topological polaritons change markedly in nature-from hyperbolic (open) to elliptical (closed) - when the integer number of crossing points $N_{A C P}$ changes from 2 to 4 , as shown in Figs. 4(b)-4(f). In other words, at the twist angle at which the topological transition arises, the number of crossing points must necessarily reach 4 , as its topology changes from open to closed.

Moreover, since $p$ polarized evanescent waves have a main contribution in the radiation heat transfer of graphene, we calculate the reflection coefficients of the $p$ polarized evanescent waves of the twisted hyperbolic system to clearly exhibit these topological transitions $[21,42]$. The imaginary part of the reflection coefficients is shown in Fig. 5. For the bilayer system without rotation in Fig. 5(a), the hyperbolic distribution of reflection coefficients is also presented, which agrees well with the bright branch of PTC and dispersion relation. We see that, when the rotation angle changes from $0^{\circ}$ to $90^{\circ}$, the imaginary part of the reflection coefficients changes from hyperbolic to elliptic, experiencing a topological transition, which is seen in the field distribution from the reflection coefficients. This means that the SPPs dispersion relations for the twisted hyperbolic system are well predicted by the reflection coefficients.

To explain visually the topological hybridization of HSPPs and the blueshift of spectral RHF, we illustratively show the dispersion and the topological transition regions as a function of rotation angles in Fig. 6. As mentioned above, the number of crossing points as a function of the angle can powerfully predict the topological transition. Nevertheless, the number of crossing points does not show the range of transitional angles where topological transitions occur. Thus, here we utilize the open angle $\psi_{\text {open }}$ of the hyperbolic branches of the individual graphene grating of the bilayer system to predict the transitional angles where topological transitions occur. For the single graphene grating of the bilayer system, hyperbolic SPPs propagate at the open angle $a \tan \left[\operatorname{Im}\left(\sigma_{y y}\right) / \operatorname{Im}\left(\sigma_{x x}\right)\right][28]$. For the twisted hyperbolic system, the two hyperbolic bands of the individual layers hybridize and strongly couple to each other at the points in wave-vector space at which they cross, leading to crossing and topological transitions. Since the hyperbolic curve is open, ideally, there is no limit on the density of states (DOS) [33]. Thus, ideally, when the open angle is paired together with a given rotation angle, that is, $\varphi_{\text {ideal }}=$ $180^{\circ}-2 \psi_{\text {open }}$, an anticrossing arises at the intersection of the bands, which may be easily predicted using geometrical arguments. However, practically, the hyperbolic SPPs will not hold when the wave vector goes to infinity due to the existence of the attenuation length. Therefore, the angle $\varphi_{\text {real }}$ at which the actual topological transition occurs lags behind the ideal 


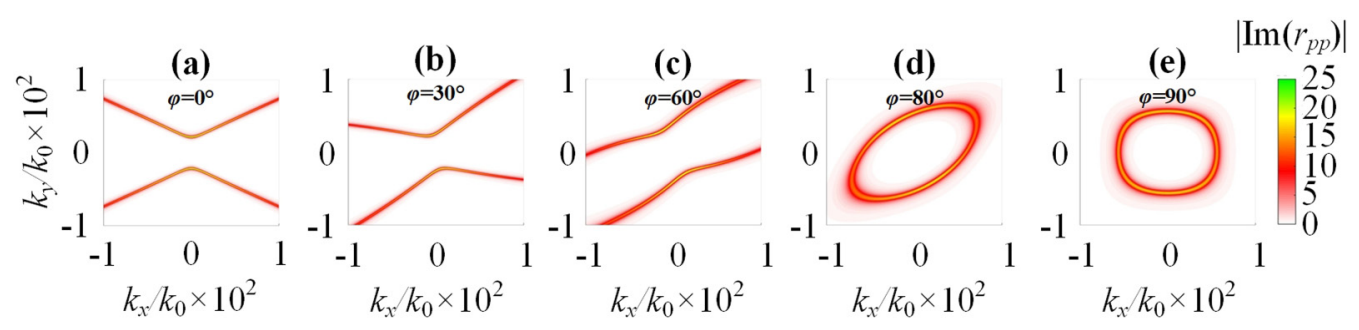

FIG. 5. Imaginary part of the reflection coefficients $r_{p p}\left(k_{x}, k_{y}\right)$ of the twisted hyperbolic system for (a) $\varphi=0^{\circ}$, (b) $\varphi=30^{\circ}$, (c) $\varphi=60^{\circ}$, (d) $\varphi=80^{\circ}$, and (e) $\varphi=90^{\circ}$ at a frequency of $0.15 \mathrm{eV} / \hbar$. The parameters are $d_{s}=1 \mathrm{~nm}, \mu=0.4 \mathrm{eV}, W=5.9 \mathrm{~nm}, P=9.84 \mathrm{~nm}$, and $\varepsilon_{s}=1$.

angle $\varphi_{\text {ideal }}$. For example, as shown in Fig. 6(a), we can see that for smaller rotation angles $\left(\varphi<36^{\circ}\right)$ there will only be a crossing in the fourth quadrant (green circular mark) and the complementary points in the second quadrant (not shown for simplicity). For larger angles $\left(\varphi>36^{\circ}\right)$, two additional crossing points emerge in the first quadrant (red circular mark) and the complementary points in the third quadrant (not shown). However, according to a frequency of $0.07 \mathrm{eV} / \hbar$ with an open angle of $76.8^{\circ}$, the ideal transitional angle $\varphi_{\text {ideal }}$ only is $26.4^{\circ}$, though the practical transitional angle $\varphi_{\text {real }}$ is much larger than the ideal transitional angle $\varphi_{\text {ideal }}$.

As the frequency increases, the open angle gradually reveals a downward trend, so the rotation angle required for the topological transition gradually becomes larger. For example, as shown in Fig. 6(b), there is only a crossing point in the second and fourth quadrants at the larger range of rotation angle $\left(0<\varphi<73^{\circ}\right)$. Meanwhile, it can be clearly seen that at a higher frequency, since the hyperbolic SPPs at a large wave vector range is easily filtered by a vacuum gap, the dif-
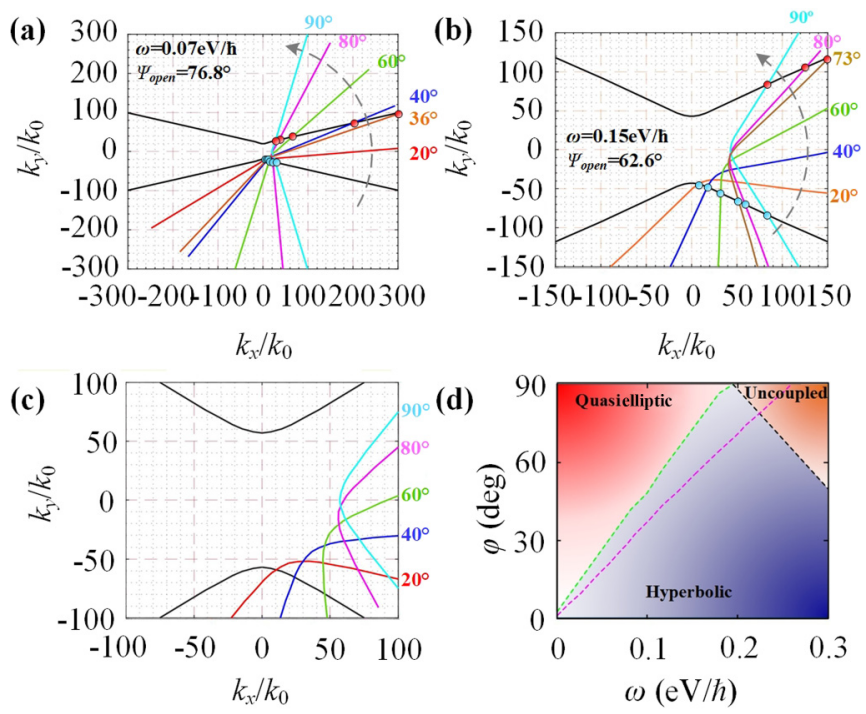

FIG. 6. Number of anticrossing points versus the angle at the frequency of (a) $0.07 \mathrm{eV} / \hbar$, (b) $0.15 \mathrm{eV} / \hbar$, and (c) $0.2 \mathrm{eV} / \hbar$. The green circular mark indicates the location of the crossing in the fourth quadrant and the red circular mark indicates the crossing point in the first quadrant. The gray arrows show the evolution of the rotation angle. (d) Topological transition regions as a function of frequency and rotation angles. The green dashed line represents the realistic topological transition angle and the pink dashed line represents the ideal ones $\left(\varphi_{\text {ideal }}=180^{\circ}-2 \psi_{\text {open }}\right)$. ference between the ideal transitional angles of the topological transition and the actual one is increased compared to the low frequency case, as shown in Fig. 6(d). This also leads to the fact that, with the further increase of frequency, the number of crossing points can no longer be up to 4 due to the finite state density in Fig. 6(c). On the other hand, as shown in Fig. 6(d), since the two hyperbolic bands of the individual layers do not couple to each other at the points in reciprocal space at which they cross, the topological transition of photonic transmission coefficient are not generated at the higher rotation angle, in turn, forming the uncoupled surface state. This uncoupled surface state would severely inhibit NFRHT. Moreover, according to Fig. 4, when the topological transition occurs, the brightest and strongest bright branch of the surface state would be produced. As shown in Fig. 6(d), the frequency of topological transition also increases with the increasing rotation angle. Thus, in Fig. 3(b), the peak of energy transfer function $\Phi(\omega)$ excited by topological transitions shows a clear blueshift.

\section{HYPERBOLIC HYBRIDIZATION DEPENDENCE OF THE HEAT TRANSFER FOR DIFFERENT VACUUM GAPS}

In order to explore the effect of twisted hybridization on heat transfer at different vacuum gap, in Fig. 7(a), we calculate the RHF as a function of rotation angle for different vacuum gaps. The curves with different colors represent different vacuum gaps. As is well known, in the field of lower vacuum gap, the RHFs of a twisted hyperbolic system in a different rotation angle can be higher than the RHF at the higher vacuum gap.

We can see in Fig. 7(a) that the presence of the rotation angle significantly modifies the RHF and can enhances the RHF of a system compared with the case without rotation angle, both for different vacuum gaps. In the cases of a smaller gap, i.e., a vacuum gap less than $100 \mathrm{~nm}$, we find that the RHF exhibits a nonmonotonic dependency versus the rotation angle, especially for a lower vacuum gap. For a small vacuum gap with $d_{0}=50 \mathrm{~nm}$, we find that the nonmonotonic trend is strong and the maximum of RHF is at a middle angle of $\varphi_{\max }=40^{\circ}$, and the RHF decreases drastically with a further increase of $\varphi$. However, for a higher vacuum gap, the nonmonotonic trend is much weaker and the rotation angle corresponding to the maximum of RHF is larger. When the vacuum gap reaches $100 \mathrm{~nm}$, compared with the nonmonotonic dependency on the rotation angle at lower vacuum gap, the larger angle can further enhance the NFRHT.

To interpret the underlying physics of the transformation of effect of twisted hybridization on heat transfer at different 

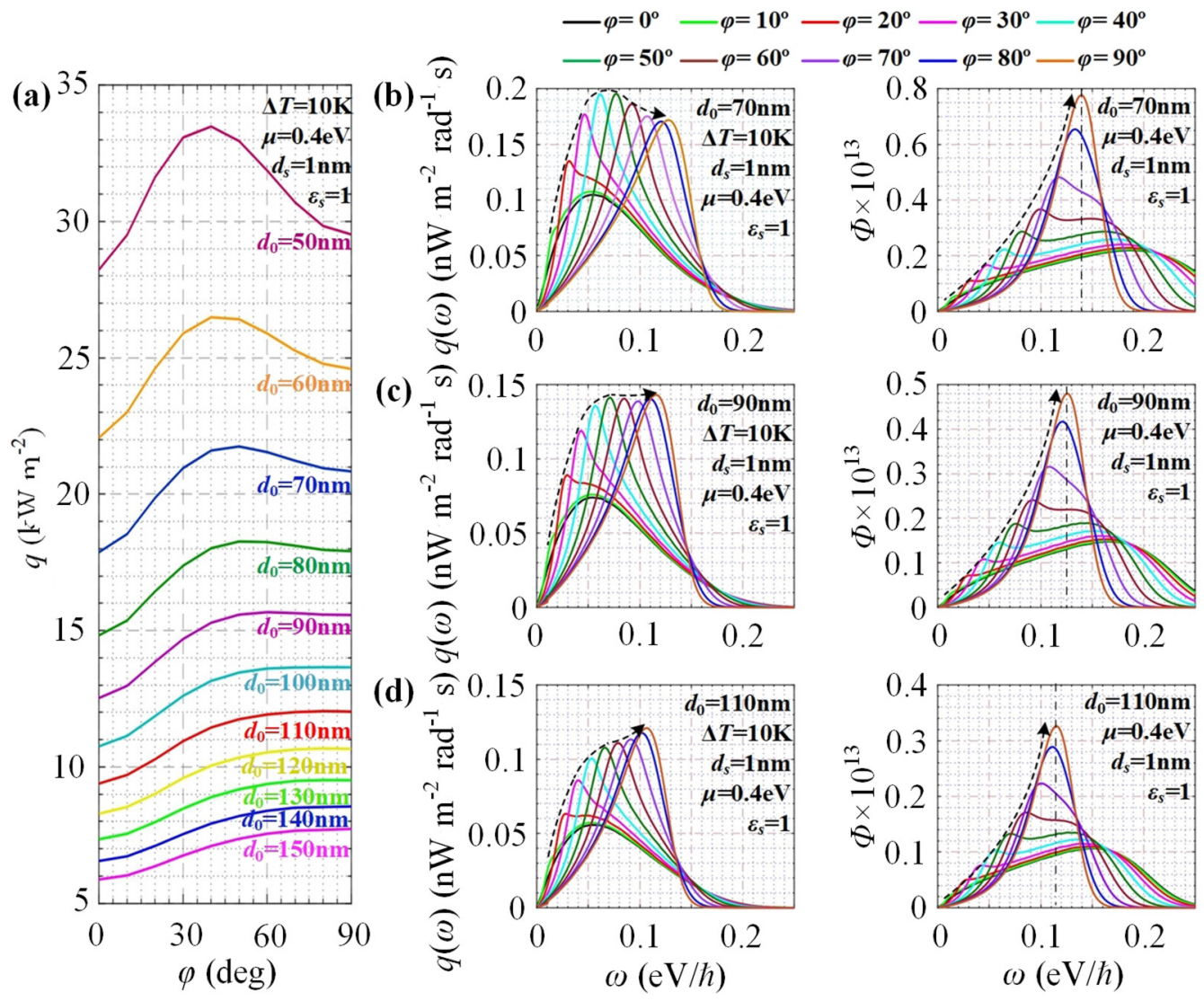

FIG. 7. (a) Radiative heat flux $q$ as a function of rotation angle $\varphi$ at different vacuum gap $d_{0}$. Spectral RHF as a function of the frequency with different rotation angle for a vacuum gap of (b) $70 \mathrm{~nm}$, (c) $90 \mathrm{~nm}$, and (d) $110 \mathrm{~nm}$. Contour plots of energy transfer function $\Phi(\omega)$ as a function of frequency with different rotation angles for a vacuum gap of (e) $70 \mathrm{~nm}$, (f) $90 \mathrm{~nm}$, and (g) $110 \mathrm{~nm}$. The parameters are $d_{s}=1 \mathrm{~nm}$, $T_{1}=310 \mathrm{~K}, T_{2}=300 \mathrm{~K}, \mu=0.4 \mathrm{eV}, W=5.9 \mathrm{~nm}$, and $P=9.84 \mathrm{~nm}$.

vacuum gaps, we show in Figs. 7(b)-7(d) the spectral RHF with different rotation angles. Now we show three gaps, namely $d_{0}=70,90$, and $110 \mathrm{~nm}$, respectively. Physically, one can expect that the different vacuum gap would bring a different spectral response. For the vacuum gap of $70 \mathrm{~nm}$, notice also that the maximum of the spectral RHF also increases drastically with the rotation angle, reaching a maximum of $0.195 \mathrm{nW} \mathrm{m}^{-2} \mathrm{rad}^{-1} \mathrm{~s}$ at $\varphi=50^{\circ}$. As the rotation angle further increases, the maximum of the spectral RHF gradually decreases to the value of $0.128 \mathrm{nW} \mathrm{m}^{-2} \mathrm{rad}^{-1} \mathrm{~s}$ at $\varphi=90^{\circ}$. As the vacuum gap increases to $90 \mathrm{~nm}$ and the rotation angle is greater than $50^{\circ}$, the peak of the spectral RHF is no longer modulated by the rotation angle, that is, the peak of the spectral RHF remains unchanged with an increase of rotation angle. In the plot of spectral RHF [Fig. 7(d)], we observe that for $d_{0}=110 \mathrm{~nm}$, the maximum of the spectral RHF is enhanced upon increasing the rotation angle, hence increasing the RHF as depicted in Fig. 7(a). To give further insight on the underlying physics, we show a plot of energy transfer function $\Phi(\omega)$ in Figs. 7(e)-7(g). When we give a larger $d_{0}$ between these bilayer structures along the $z$ axis, a spectral redshift may be observed for the same rotation phase. Namely, as the vacuum gap increases, the frequency of topological transitions of different rotation angles would be lower. We stress that, in addition to the twisted effects inside the bilayer structure the mean energy of a Planck oscillator would play a significant role on the spectral RHF. For a lower frequency, it would also gradually amplify the contribution of the increase of energy transfer function generated by topological transition to the enhancement of NFRHT. The spectral redshift generated by increasing the vacuum gap fortunately allows people to track intuitively the increase of the energy transfer function at spectral RHF. Therefore, it can be clearly observed that with the increase of the vacuum gap, the spectral peak and the rotation angle show a positive correlation in Figs. 7(b)-7(d).

As the vacuum gap increases, the frequency of topological transitions of rotation angles would be lower, which also means that the angle of topological transitions occuring at fixed frequencies become bigger. To visually reveal the physics, as shown in Figs. 8(a)-8(c), we plot the PTC, the dispersion relation, and the corresponding rotation of the optical axis. The top row shows the PTCs without rotation for the vacuum gap of 70,90 , and $110 \mathrm{~nm}$, respectively. It can be distinctly observed that due to the existence of the attenuation length, the wave-vector range of surface state drastically shrinks with increase of the vacuum gap. When the vacuum gap of the system increases from 70 to $90 \mathrm{~nm}$, the maximum wave vector of the bright branches in the positive $k_{x}$ quadrant significantly decreases from $90 k_{0}$ to $70 k_{0}$. At the vacuum gap of $110 \mathrm{~nm}$, the maximum wave vector of the bright branches in the positive $k_{x}$ quadrant only maintain $50 k_{0}$. Thus, in the bottom row of Figs. 8(a)-8(c) showing the PTCs 


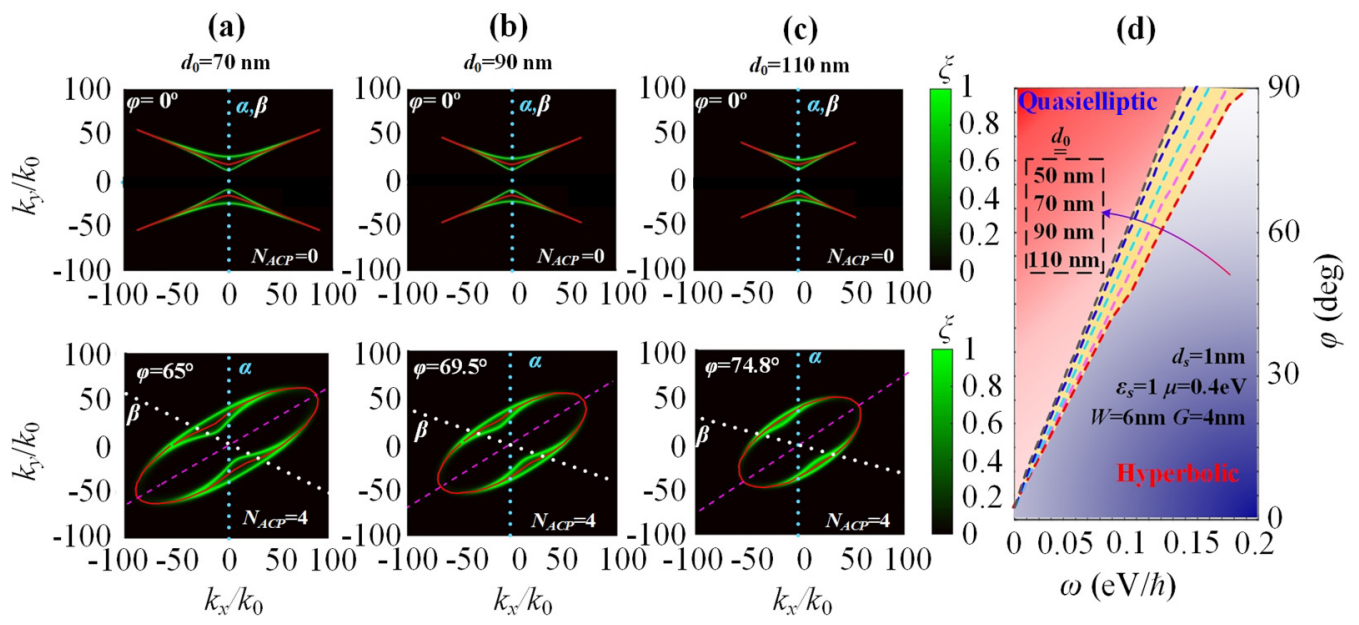

FIG. 8. (a)-(c) The top row shows the numerically simulated PTCs without rotation at the vacuum gap of 70, 90, and $110 \mathrm{~nm}$ at a frequency of $0.12 \mathrm{eV} / \hbar$, respectively. The bottom row shows the PTCs at the rotation angle of topological transitions for the vacuum gap of 70,90 , and $110 \mathrm{~nm}$, respectively. The red solid lines are the dispersion after hybridization of twisted hyperbolic system. The pink dashed lines are the dispersion after hybridization of twisted hyperbolic system. The blue dotted lines and the dotted dashed lines are the optical axis of the top grating (no rotation) and bottom grating (with rotation) of twisted bilayer system. (d) Topological transition regions as a function of frequency and rotation angles at different vacuum gap.

at the rotation angle of topological transitions, the surface state of HSPPs with a smaller wave-vector range need to rotate a larger angle to complete the topological transition with an increase of the vacuum gap. These results also directly exhibit the delayed effect that the system at a larger vacuum gap needs a larger transitional angle of topological transitions. In order to more clearly observe the transition of the surface state of the system from hyperbolic to elliptical at a different vacuum gap, we plot the topological transition regions as a function of frequency and rotation angles at different vacuum gaps in Fig. 8(d). The dashed lines with different colors represent the topological transition angle with different vacuum gaps. In Fig. 8(d), a larger vacuum gap would push the topological transition angle at different frequencies to a higher range. In other words, a larger vacuum gap would make the topological transition frequency of different rotation angles redshifted, especially for systems with large rotation angles. These results also explain the redshift of the spectral RHF and the energy transfer function with increasing of vacuum gap in Fig. 7.

\section{HYBRIDIZATION EFFECT FOR DIFFERENT THICKNESSES OF DIELECTRIC SPACER}

Note that one important aspect of the twisted hybridization is the dependence of the thickness of the dielectric spacer between adjacent gratings in the bilayer. As shown in Eq. (A9), the interbedded coupling strength at the bilayer is essentially dependent on the distance between two hyperbolic polaritons. More generally, the term $e^{-i k_{z 0} d_{s}}$ determines the strength of the coupling. Thus, the twisted hybridization of hyperbolic polaritons can be affected by the thickness of the spacer between adjacent sheets in the bilayer.

To get insight into the role of the thickness of the dielectric spacer on the NFRHT of the twisted hyperbolic system we show the radiative heat flux $q$ as a function of the thickness at $d_{0}=50 \mathrm{~nm}$ for different rotation angles $\varphi$ in Fig. 9. As shown in Fig. 9(a), we find that the RHF without rotation exhibits a nonmonotonic dependency versus the separated thickness. In fact, we find that the very compact bilayer structure reveals a suppressed heat transfer compared with the one with larger thickness. A local maximum of heat transfer is observed as the $d_{0}$ is comparable but a little bit larger than $d_{s}$. After the

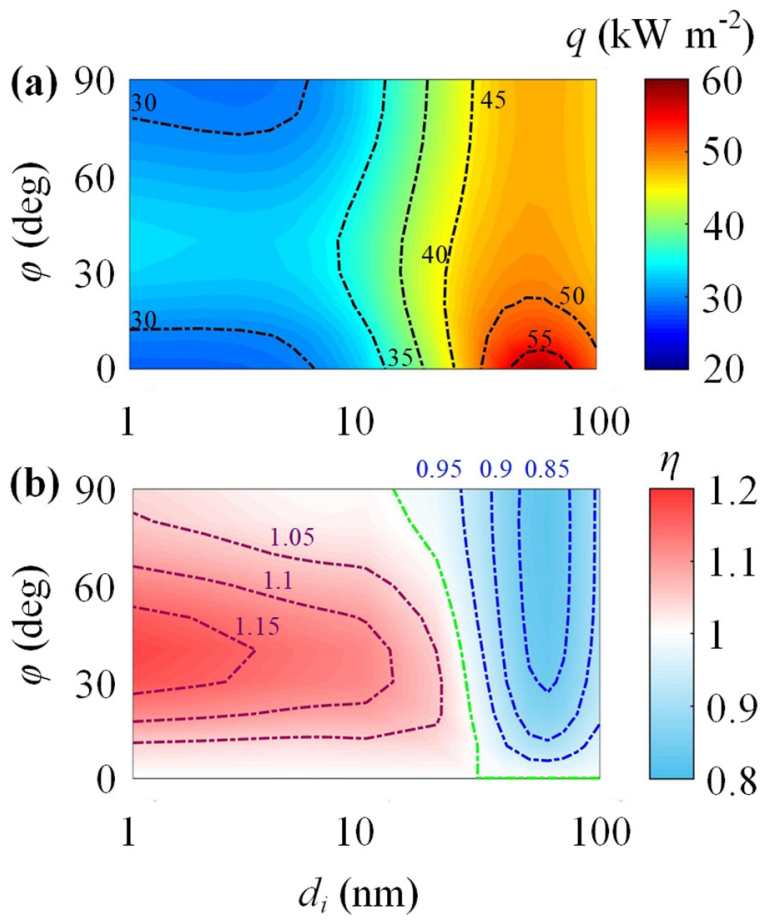

FIG. 9. (a) Radiative heat flux $q$ as a function of separated thickness $d_{s}$ at different rotation angles $\varphi$. (b) Heat transfer coefficient ratio $\eta$ between hyperbolic system and nonrotating system as a function of separated thickness and rotation angle. The parameters are $d_{0}=50 \mathrm{~nm}, T_{1}=310 \mathrm{~K}, T_{2}=300 \mathrm{~K}, \mu=0.4 \mathrm{eV}, W=5.9 \mathrm{~nm}$, and $P=9.84 \mathrm{~nm}$. 

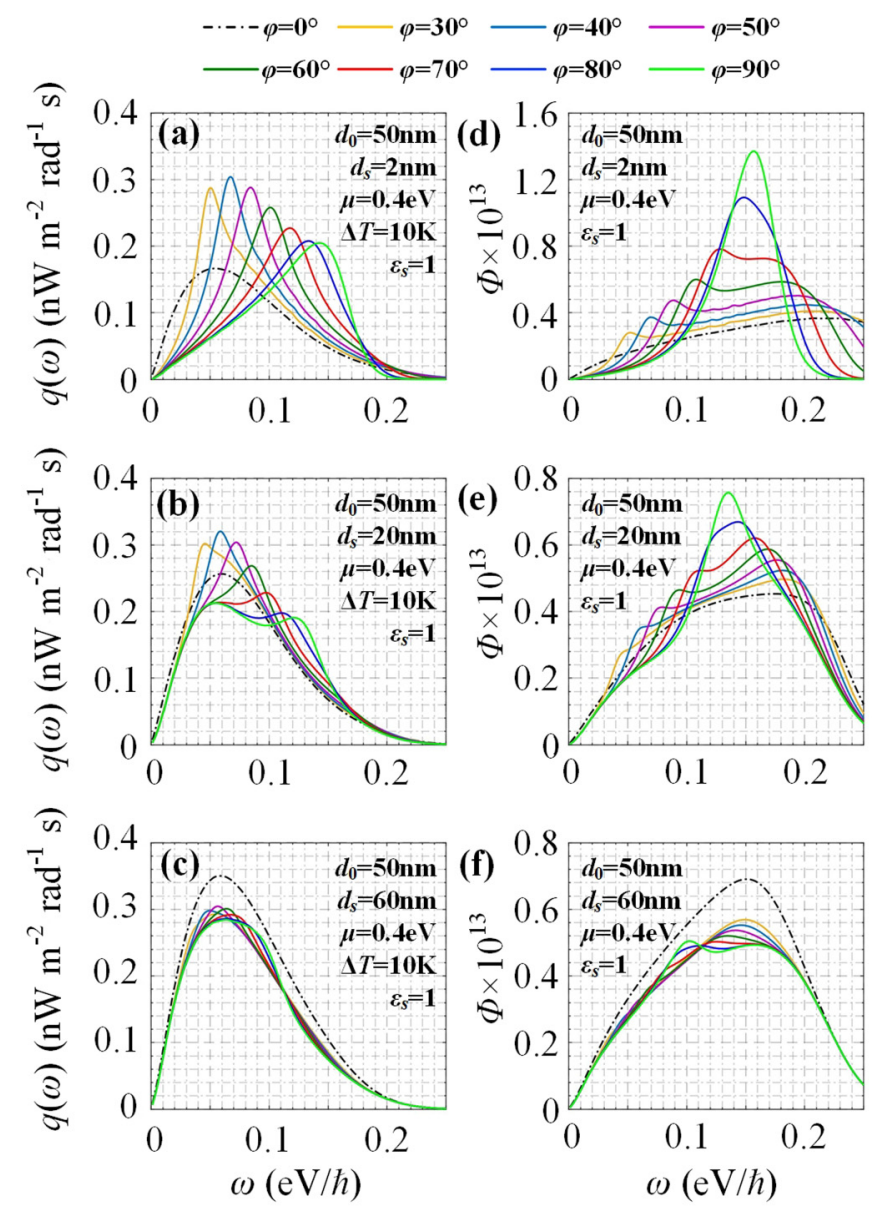

FIG. 10. Spectral RHF as a function of the frequency with different rotation angles for the thickness of dielectric spacer of (a) $2 \mathrm{~nm}$, (b) $20 \mathrm{~nm}$, and (c) $60 \mathrm{~nm}$. Contour plots of energy transfer function $\Phi(\omega)$ as a function of frequency with different rotation angles for a vacuum gap for the thickness of dielectric spacer of (d) $2 \mathrm{~nm}$, (e) $20 \mathrm{~nm}$, and (f) $60 \mathrm{~nm}$. The parameters are $d_{0}=50 \mathrm{~nm}, T_{1}=310 \mathrm{~K}$, $T_{2}=300 \mathrm{~K}, \mu=0.4 \mathrm{eV}, W=5.9 \mathrm{~nm}$, and $P=9.84 \mathrm{~nm}$.

local maximum point, $q$ decreases, because as the thickness increases, the evanescent wave from the second graphene grating is gradually filtered by the vacuum gap. In order to give a more intuitive feeling of the effect on the RHF from the twisted hyperbolic system with different separated thicknesses, Fig. 9(b) presents the RHF ratio $\eta$ between the twisted hyperbolic system and a nonrotating system as a function of separated thickness and rotation angle at $d_{0}=50 \mathrm{~nm}$. We see that as $d_{s} \leqq 10 \mathrm{~nm}$, the ratio first increases and then decreases with increasing rotation angle. When the separated thickness is $1 \mathrm{~nm}$, the maximum heat transfer coefficient ratio $\eta$ can be close to 1.2. The strongest suppression occurs when the thickness is $60 \mathrm{~nm}$, which the maximum suppression can be close to 0.8 .

Now, we turn to the near-field spectral radiative heat flux. Figures 10(a)-10(c) show the spectra of radiative heat flux under different rotation angles for the thickness of 2, 20, and $60 \mathrm{~nm}$, respectively. As shown in Figs. 10(a)-10(c), in the absence of rotation, we note that the contribution from the thickness of the dielectric spacer to amplify the spectral RHF is gradually stronger. When the thickness of dielectric spacer reaches $2 \mathrm{~nm}$ from $60 \mathrm{~nm}$, the maxima of the spectral RHF is increased from $0.17 \mathrm{nW} \mathrm{m}^{-2} \mathrm{rad}^{-1} \mathrm{~s}$ to around $0.35 \mathrm{nW} \mathrm{m}^{-2} \mathrm{rad}^{-1} \mathrm{~s}$. However, this obvious enhancement effect on the spectral RHF can be ignored at a smaller rotation angle $\left(\phi<50^{\circ}\right)$ in Figs. 10(b) and 10(c). This also leads to that, for the system with a larger thickness of dielectric spacer, the increase in the RHF supported by rotation is gradually reduced, ultimately producing the suppression effect in Fig. 9. Compared with the system with smaller rotation angle, a larger thickness of dielectric spacer would lead to the obvious redshift and increasing of the spectral RHF after the rotation angle exceeds $50^{\circ}$. To get insight into the role of the rotation angle on the spectra of radiative heat flux with different thicknesses of dielectric spacer, we calculate the energy transfer function in Figs. 10(d)-10(f). As a thickness of dielectric spacer with $d_{s}=20 \mathrm{~nm}$ is used, we see in Fig. 10(e) that the energy transfer function gets broader but the maximum decreases compared with the ones at $d_{s}=2 \mathrm{~nm}$, especially in $\phi=90^{\circ}$. This trend is particularly obvious when the thickness of dielectric spacer increases to $60 \mathrm{~nm}$. Meanwhile, it can be clearly seen that the influence of the thickness of the dielectric spacer on the energy transfer coefficient at small rotation angles $\left(\phi<50^{\circ}\right)$ and low frequencies is almost negligible. As a result, the effect for the thickness of the dielectric spacer on spectral RHF is very limited at a smaller rotation angle $(\phi<$ $\left.50^{\circ}\right)$ in Figs. 10(b) and 10(c). For larger rotation angle $(\phi>$ $50^{\circ}$ ), the redshift caused by the increase in thickness results in the system occupying a higher energy transfer coefficient at low frequencies, which in turn leads to the spectral RHF redshift and increases in Figs. 10(b) and 10(c). Meanwhile, as the thickness of dielectric spacer increases, the enhancement effect of twisted hyperbolic system on the energy transfer function gradually decreases. As shown in Fig. 10(b), when the rotation angle of the system at $d_{s}=2 \mathrm{~nm}$ is increased from $0^{\circ}$ to $90^{\circ}$, the energy transfer function at a frequency of $0.13 \mathrm{eV} / \hbar$ significantly increases from $0.31 \times 10^{13}$ to $0.94 \times$ $10^{13}$. However, at the thickness of $20 \mathrm{~nm}$, the energy transfer function at a frequency of $0.13 \mathrm{eV} / \hbar$ increases from $0.44 \times$ $10^{13}$ to $0.75 \times 10^{13}$. Moreover, for the thickness of $60 \mathrm{~nm}$, the energy transfer function at a frequency of $0.13 \mathrm{eV} / \hbar$ begins to attenuate from $0.65 \times 10^{13}$ to $0.48 \times 10^{13}$.

The origin of the dependency of the thickness of dielectric spacer on HSPPs supported by the twisted hyperbolic system can be understood with a concrete analysis of the energy transmission coefficient and the dispersion relations. In particular, we show the PTC with a thickness of dielectric spacer of 2, 20, and $60 \mathrm{~nm}$, respectively, in Fig. 11. A frequency of $0.13 \mathrm{eV} / \hbar$ and $\phi=0^{\circ}$ is considered for the left figures and a frequency of $0.13 \mathrm{eV} / \hbar$ and $\phi=0^{\circ}$ for the right figures. Meanwhile, we plot the plasmon dispersion relations in Fig. 11 where the white curves represent the plasmon dispersions. When the two bodies are brought into proximity, the evanescent field of HSPPs associated with two graphene gratings can interact with each other, leading to the two dispersion curves at the higher wave-vector region (symmetrical dispersion) and the smaller wave-vector region (antisymmetrical dispersion) $[48,49]$. For $\phi=0^{\circ}$, as the dielectric spacer becomes thicker, the HSPP's bright band supported by symmetrical dispersion gradually shifts to the low wave-vector area, resulting in a gradual decrease in the dissipation of the vacuum gap. As a 


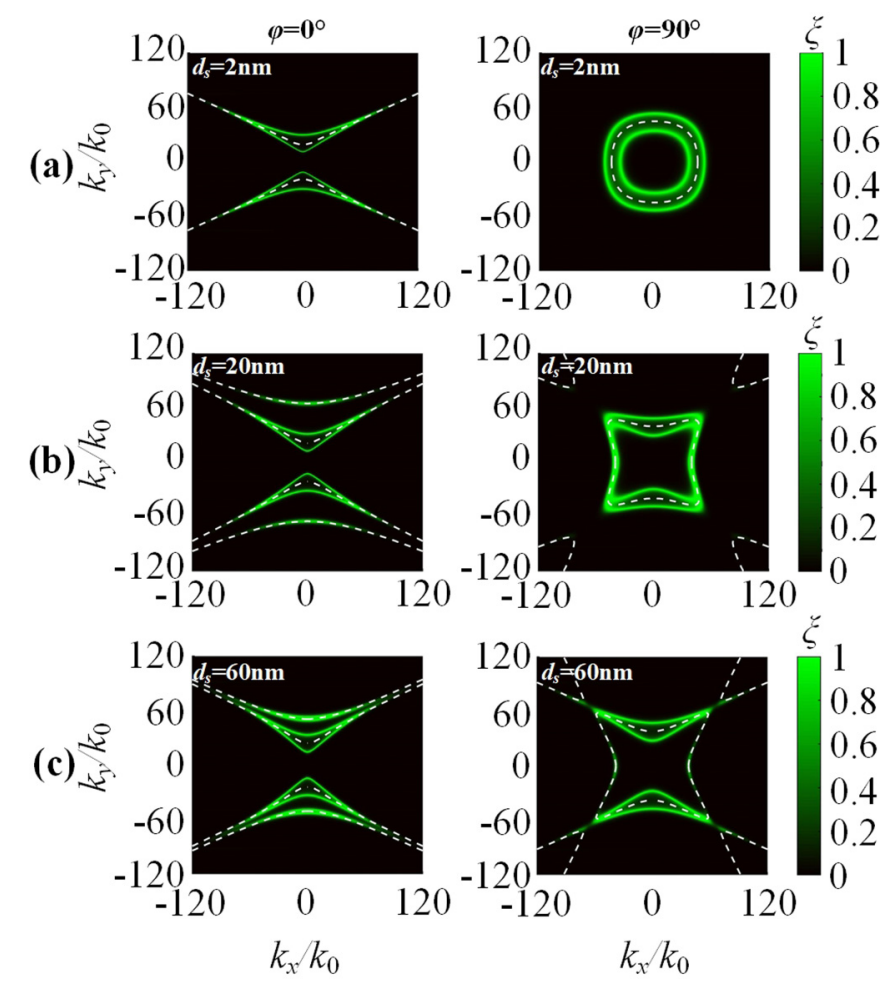

FIG. 11. (a)-(c) The left row shows the numerically simulated PTCs without rotation at the thickness of dielectric spacer of 2, 20, and $60 \mathrm{~nm}$, respectively. The right row shows the PTCs at the rotation angle of $90^{\circ}$ for the thickness of dielectric spacer of 2, 20, and 60 $\mathrm{nm}$, respectively. The white dashed lines are the dispersion after hybridization of hyperbolic system.

result, the SPPs bright band supported by symmetrical dispersion gradually becomes brighter as its contribution to energy transfer gradually increases. Consequently, in the absence of rotation angle, we obtain a large RHF for a thicker dielectric spacer, as shown in Figs. 9 and 10. We can see that, for $\phi=90^{\circ}$ with the increasing of thickness of the dielectric spacer, the shape of the SPP's bright band and dispersion relations begins to change significantly, that is, it changes from an isotropic circle to an anisotropic rhombus. Meanwhile, for $\phi=90^{\circ}$, due to the coupling strength will decay when the thickness increases, the strength of the SPP's bright band supported by a second layer of graphene grating at the bilayer system becomes weaker, which in turn leads to the enhancement effect of the twisted hyperbolic system on the energy transfer function gradually decreasing in Fig. 10. This is because for a large separation, two graphene gratings would be very weakly coupled and the anticrossing feature will resultantly be very weak and nearly disappear. It can be observed that the surface state degrades into the hyperbolic bright band due to the surface state supported by the edge of the rhombus dispersion perpendicular to the $k_{x}$ axis being already very weak.

To show thoroughly these effects, Fig. 12 shows the isofrequency dispersion curves for a superlattice considering the thickness of dielectric spacer at the frequency of $0.13 \mathrm{eV} / \hbar$. In the thickness of dielectric spacer at $d_{s}=2 \mathrm{~nm}$, since all supported SPPs exhibit identical characteristics independently

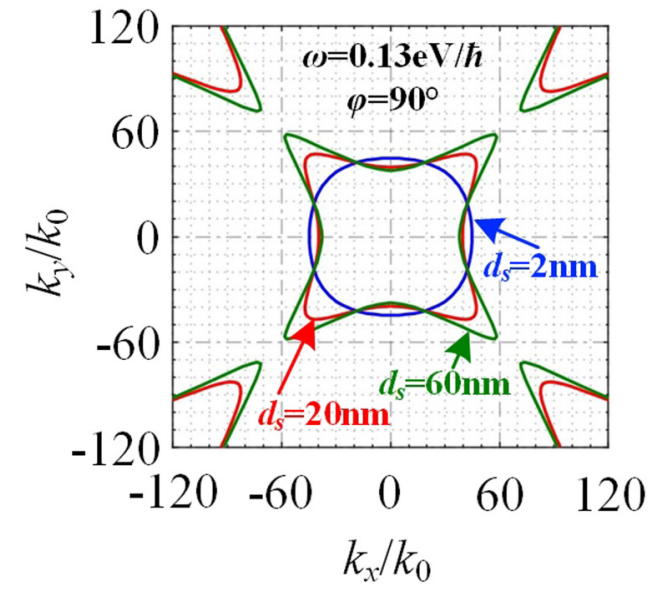

FIG. 12. Dispersion dependence on the thickness of dielectric spacer at the frequency of $0.13 \mathrm{eV} / \hbar$. Thickness essentially determines the coupling strength. Here, the mutual angle is $90^{\circ}$.

of their propagation direction, the SPP is isotropic and thus possesses a circular IFC. As the thickness of dielectric spacer increases, due to two evanescently coupled polaritons with respect to one another in the bilayer system, the shape of the IFC gradually changes from circular to quasirhombus. With a thicker dielectric spacer, the superlattice supports higher $k$ surface states. Meanwhile, as seen in in Fig. 12, the hyperbolic branches in the corners are visible and move towards the low wave-vector area with increasing thickness of dielectric spacer.

\section{CONCLUSIONS}

In summary, the present work revealed topological transition of plasmon polaritons in near-field radiative heat transfer between twisted hyperbolic systems composed of a bilayer of twisted graphene gratings. As the rotation angle increases, the surface states of the twisted hyperbolic systems gradually change from open (hyperbolic) to closed (elliptical) contours. Meanwhile, as the topological transition of plasmon polaritons gradually appears, the surface state becomes more intense, resulting an increase in near-field radiative heat transfer. The underlying physics are interpreted qualitatively through analyzing the dispersion of the individual metasurfaces in the wave-vector space as well as peculiar anticrossing features linked by the topological nature of the number of anticrossing points, clearly highlighting the role played by the rotation angle. Moreover, we have found that due to the dissipative characteristics of evanescent waves in vacuum, the transitional angle of topological transition lags behind the angle predicted by the open angle of the independent hyperbolic branches. This hysteresis effect of topological transitions would be more obvious with increasing vacuum gap. Finally, we find the transition from the enhancement effect of heat transfer caused by the twisted hyperbolic system to a suppression one with increasing thickness of dielectric spacer. Interestingly, the thickness of the dielectric spacer would distinctly modify the shape of topological polaritons. For example, at a rotation angle of $90^{\circ}$, there exists a mode of evolution of topological polaritons with different thicknesses 
of dielectric space (from circular to quasirhombus) due to the change of the anticrossing feature.

Our work represents a first step in the study of the modification of energy exchanges mediated by twisted physics and provides a hybridization effect of polaritons on near-field radiation heat transfer.

\section{ACKNOWLEDGMENTS}

This work was supported by the National Natural Science Foundation of China (Grant No. 51706053) and by the Fundamental Research Funds for the Central Universities (Grant No. AUGA5710094020).

\section{APPENDIX A: REFLECTION MATRIX R OF IN MULTILAYER ANISOTROPIC METASURFACES}

In this appendix, a generalized $4 \times 4 T$-matrix formalism for arbitrary anisotropic 2D layers is developed, from which the general relations for the surface waves dispersions and reflection matrix $\mathbf{R}$ are deduced. Let us first consider a single anisotropic metasurface at the interface between two semiinfinite media. Within the homogenization approach, the EM response of such a metasurface, in general, can be described by a fully populated conductivity tensor $\hat{\sigma}^{\prime \prime}$ in the wave-vector space. Besides, $\hat{\sigma}^{\prime \prime}$ denotes the conductivity tensor in the wave-vector space [50],

$$
\hat{\sigma}^{\prime \prime}=\left(\begin{array}{ll}
\sigma^{\prime \prime}{ }_{x x} & \sigma^{\prime \prime}{ }_{x y} \\
\sigma^{\prime \prime}{ }_{y x} & \sigma^{\prime \prime}{ }_{y y}
\end{array}\right)=\frac{1}{k^{2}}\left(\begin{array}{ll}
k_{x}^{2} \sigma_{x x}+k_{y}^{2} \sigma_{y y}+k_{x} k_{y}\left(\sigma_{x y}+\sigma_{y x}\right) & k_{x}^{2} \sigma_{x y}-k_{y}^{2} \sigma_{y x}+k_{x} k_{y}\left(\sigma_{y y}-\sigma_{x x}\right) \\
k_{x}^{2} \sigma_{y x}-k_{y}^{2} \sigma_{x y}+k_{x} k_{y}\left(\sigma_{y y}-\sigma_{x x}\right) & k_{x}^{2} \sigma_{y y}+k_{y}^{2} \sigma_{x x}-k_{x} k_{y}\left(\sigma_{x y}+\sigma_{y x}\right)
\end{array}\right) .
$$

Following Refs. [51-53] let us write separately the EM field of the $p$-polarized (TM) and $s$-polarized (TE) components of the EM wave, which then will be mixed by the nondiagonal response of a metasurface. The $p$ waves with the magnetic field perpendicular to the plane of incidence possess the EM field components $E_{p}=\left\{E_{x}, 0, E_{z}\right\}, \quad H_{p}=$ $\left\{0, H_{y}, 0\right\}$. For the $s$ waves, with the electric field perpendicular to the plane of incidence, the EM field components are $E_{s}=\left\{0, E_{y}, 0\right\}, H_{s}=\left\{H_{x}, 0, H_{z}\right\}$. Substituting $p$ wave and $s$ wave into the boundary conditions of the metasurface [54], one obtain the $4 \times 4 T$ matrix, which gives the relation between all the electric field components in the media above and below the metasurface:

$$
\left[\begin{array}{c}
H_{p 1}^{+} \\
H_{p 1}^{-} \\
E_{\mathrm{s} 1}^{+} \\
E_{\mathrm{s} 1}^{-}
\end{array}\right]=\widehat{T}_{1 \rightarrow 2}\left[\begin{array}{c}
H_{p 2}^{+} \\
H_{p 2}^{-} \\
E_{\mathrm{s} 2}^{+} \\
E_{\mathrm{s} 2}^{-}
\end{array}\right]
$$

here, the signs + and - represent the forward and backward waves. $\widehat{T}_{1 \rightarrow 2}$ can be derived as

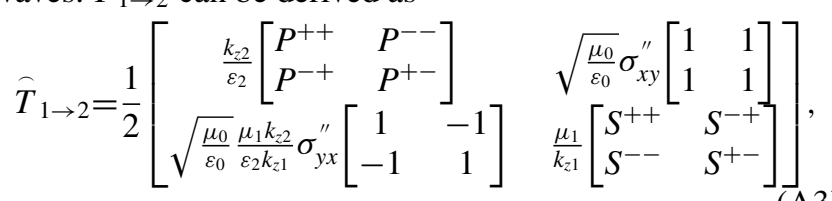

where the $p$-wave components are

$$
\begin{aligned}
& P^{++}=\frac{\varepsilon_{1}}{k_{z 1}}+\frac{\varepsilon_{2}}{k_{z 2}}+\frac{\sigma_{x x}^{\prime \prime}}{\omega \varepsilon_{0}} ; P^{--}=-\frac{\varepsilon_{1}}{k_{z 1}}+\frac{\varepsilon_{2}}{k_{z 2}}-\frac{\sigma_{x x}^{\prime \prime}}{\omega \varepsilon_{0}}, \\
& P^{-+}=-\frac{\varepsilon_{1}}{k_{z 1}}+\frac{\varepsilon_{2}}{k_{z 2}}+\frac{\sigma_{x x}^{\prime \prime}}{\omega \varepsilon_{0}} ; P^{+-}=\frac{\varepsilon_{1}}{k_{z 1}}+\frac{\varepsilon_{2}}{k_{z 2}}-\frac{\sigma_{x x}^{\prime \prime}}{\omega \varepsilon_{0}},
\end{aligned}
$$

where the $s$-wave components are

$$
\begin{aligned}
& S^{++}=\frac{k_{z 1}}{\mu_{1}}+\frac{k_{z 2}}{\mu_{2}}+\omega \mu_{0} \sigma_{y y}^{\prime \prime} ; S^{-+}=\frac{k_{z 1}}{\mu_{1}}-\frac{k_{z 2}}{\mu_{2}}+\omega \mu_{0} \sigma_{y y}^{\prime \prime}, \\
& S^{--}=\frac{k_{z 1}}{\mu_{1}}-\frac{k_{z 2}}{\mu_{2}}-\omega \mu_{0} \sigma_{y y}^{\prime \prime} ; S^{+-}=\frac{k_{z 1}}{\mu_{1}}+\frac{k_{z 2}}{\mu_{2}}-\omega \mu_{0} \sigma_{y y}^{\prime \prime} ;
\end{aligned}
$$

here, subscripts 1 and 2 represent the media above and below the metasurface, respectively. $k_{z}, \mu$, and $\varepsilon$ are tangential wave vector along the $z$ direction in the media, the magnetic permeability of the media, and the dielectric function of the media. Subscripts $p$ and $s$ represent $p$ polarized (TM) and $s$ polarized (TE), respectively. $\varepsilon_{0}$ is the vacuum permittivity; $\mu_{0}$ is the vacuum permeability. In general, for any $4 \times 4 T$ matrix that links all the electric field components in a first layer with those in $N$ layer,

$$
\left[\begin{array}{c}
H_{p 1}^{+} \\
H_{p 1}^{-} \\
E_{\mathrm{s} 1}^{+} \\
E_{\mathrm{s} 1}^{-}
\end{array}\right]=\left[\begin{array}{llll}
T_{11} & T_{12} & T_{13} & T_{14} \\
T_{21} & T_{22} & T_{23} & T_{24} \\
T_{31} & T_{32} & T_{33} & T_{34} \\
T_{41} & T_{42} & T_{43} & T_{44}
\end{array}\right]\left[\begin{array}{c}
H_{p 2}^{+} \\
H_{p 2}^{-} \\
E_{\mathrm{s} 2}^{+} \\
E_{\mathrm{s} 2}^{-}
\end{array}\right]
$$

The reflection matrix $\mathbf{R}$ is defined and expressed in terms of the $T$-matrix elements as follows [55]:

$$
\begin{gathered}
r_{p p}=\left.\frac{H_{p 1}^{-}}{H_{p 1}^{+}}\right|_{E_{s 1}^{+}=0}=\frac{T_{21} T_{33}-T_{23} T_{31}}{T_{11} T_{33}-T_{13} T_{31}}, \\
r_{p \mathrm{~s}}=\left.\frac{E_{s 1}^{-}}{H_{p 1}^{+}}\right|_{E_{s 1}^{+}=0}=\frac{T_{41} T_{33}-T_{43} T_{31}}{T_{11} T_{33}-T_{13} T_{31}}, \\
r_{\mathrm{s} p}=\left.\frac{H_{p 1}^{-}}{E_{s 1}^{+}}\right|_{H_{p 1}^{+}=0}=\frac{T_{11} T_{23}-T_{13} T_{21}}{T_{11} T_{33}-T_{13} T_{31}}, \\
r_{\mathrm{ss}}=\left.\frac{E_{s 1}^{-}}{E_{s 1}^{+}}\right|_{H_{p 1}^{+}=0}=\frac{T_{11} T_{43}-T_{13} T_{41}}{T_{11} T_{33}-T_{13} T_{31}} .
\end{gathered}
$$

The formalism developed above can be easily generalized for an arbitrary number of layers by multiplying the $T$ matrices corresponding to each layer. For a multilayer metasurface consisting of $N$ 2D layers with effective conductivity tensors $\hat{\sigma}_{j}^{\prime \prime}(i=1,2, \ldots, N)$. One obtains the $4 \times 4 T$ matrix, which gives the relations between all the electric field components and the magnetic field components in the multilayer 

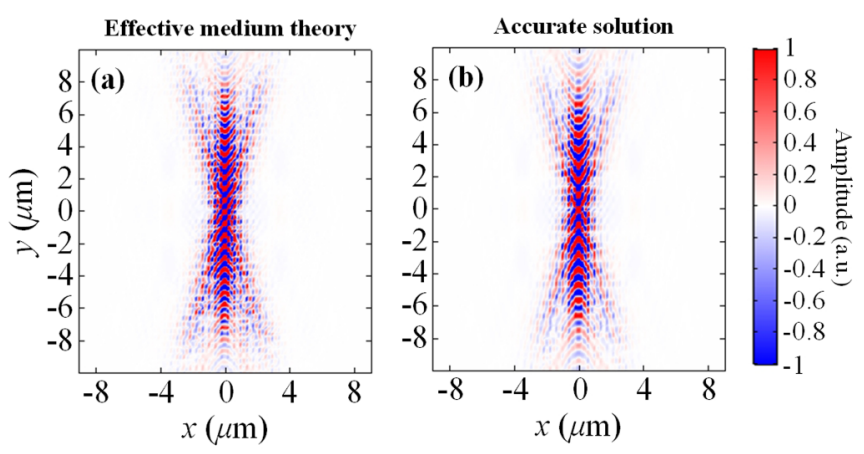

FIG. 13. E-field component of surface plasmons of bilayer graphene gratings with an interlayer distance of $1 \mathrm{~nm}$ at a frequency of $0.0021 \mathrm{eV} / \hbar$ : (a) effective medium theory, and (b) accurate solution.

metasurface:

$$
\left[\begin{array}{c}
H_{p 1}^{+} \\
H_{p 1}^{-} \\
E_{\mathrm{s} 1}^{+} \\
E_{\mathrm{s} 1}^{-}
\end{array}\right]=\hat{T}_{1 \rightarrow N}\left[\begin{array}{c}
H_{p N}^{+} \\
H_{p N}^{-} \\
E_{s N}^{+} \\
E_{s N}^{-}
\end{array}\right]
$$

here

$$
\widehat{T}_{1 \rightarrow N}=\widehat{T}_{1} \widehat{T}_{d_{i, 1}} \widehat{T}_{2} \widehat{T}_{d_{i, 2}} \ldots \widehat{T}_{d_{i, N-1}} \widehat{T}_{N}
$$

where $\widehat{T}_{j}$ is obtained from $\widehat{T}_{1}$ [Eq. (A3)] by replacing $\hat{\sigma}_{1}^{\prime \prime}$ with $\hat{\sigma}_{j}^{\prime \prime} ; \widehat{T}_{d_{i, j}}$ is the $T$ matrices for a light propagating through the vacuum between two adjacent $2 \mathrm{D}$ layers with corresponding thicknesses $d_{i, j}(j=1,2, \ldots, N-1)$ :

$$
T_{d_{i, j}}=\left[\begin{array}{cccc}
e^{-i k_{z j} d_{i, j}} & 0 & 0 & 0 \\
0 & e^{i k_{z j} d_{i, j}} & 0 & 0 \\
0 & 0 & e^{-i k_{z j} d_{i, j}} & 0 \\
0 & 0 & 0 & e^{i k_{z j} d_{i, j}}
\end{array}\right] .
$$

The dispersion of the collective surface waves in such an $N$-layer system can be found as zeros of the denominator of the reflection coefficients:

$$
T_{11} T_{33}-T_{13} T_{31}=0 .
$$

The $T$ matrix allows us to obtain all necessary characteristics (the reflection and the dispersion relation) using the general Eqs. (A3)-(A11).

\section{APPENDIX B: VERIFICATION OF EFFECTIVE MEDIUM THEORY}

In order to verify that the EMT can accurately describe the optical character of the bilayer grating, we used the COMSOL software to calculate electric field distributions based on the effective medium theory and accurate solution, respectively (see Fig. 13). We consider that the width $W$ is $24 a(5.9 \mathrm{~nm})$ and the unit-cell period $P$ is $40 a(9.84 \mathrm{~nm})$. The accurate solution of the electric field distribution is based on the accurate construction of the grating model and is calculated via COMSOL. It can be seen that, compared with the accurate solution, the EMT can describe the electric field distribution of the bilayer graphene grating very well.
[1] D. Polder and M. Van Hove, Phys. Rev. B 4, 3303 (1971).

[2] Y. Yang and L. P. Wang, Phys. Rev. Lett. 117, 044301 (2016).

[3] V. Fernández-Hurtado, F. J. García-Vidal, S. H. Fan, and J. C. Cuevas, Phys. Rev. Lett. 118, 203901 (2017).

[4] Z. M. Zhang, Nano/Microscale Heat Transfer (Springer Nature, Switzerland AG, 2020).

[5] O. Ilic, M. Jablan, J. D. Joannopoulos, I. Celanovic, H. Buljan, and M. Soljacic, Opt. Express. 20, A366 (2012).

[6] T. Koyama, T. Inoue, D. D. Kang, K. Ikeda, T. Asano, and S. Noda, Nano Lett. 19, 3948 (2019).

[7] G. R. St-Gelais, G. R. Bhatt, L. X. Zhu, S. H. Fan, and M. Lipson, ACS Nano 11, 3001 (2017).

[8] B. Zhao, K. F. Chen, S. Buddhiraju, G. Bhatt, M. Lipson, and S. H. Fan, Nano Energy 41, 344 (2017).

[9] C. R. Otey, W. T. Lau, and S. H. Fan, Phys. Rev. Lett. 104, 154301 (2010).

[10] C. L. Zhou, L. Qu, Y. Zhang, and H. L. Yi, J. Quant. Spectrosc. Radiat. Transfer 251, 107023 (2020).

[11] V. Kubytskyi, S.-A. Biehs, and P. Ben-Abdallah, Phys. Rev. Lett. 113, 074301 (2014).

[12] L. X. Zhu, A. Fiorino, D. Thompson, R. Mittapally, E. Meyhofer, and P. Reddy, Nature (London) 566, 239 (2019).

[13] M. Ghashami, H. Y. Geng, T. Kim, N. Iacopino, S. K. Cho, and K. Park, Phys. Rev. Lett. 120, 175901 (2018).

[14] L. Tang, J. DeSutter, and M. Francoeur, ACS Photonics 7, 1304 (2020).
[15] S. A. Biehs, M. Tschikin, and P. Ben-Abdallah, Phys. Rev. Lett 109, 104301 (2012).

[16] H. H. Wu, Y. Huang, L. J. Cui, and K. Y. Zhu, Phys. Rev. Appl. 11, 054020 (2019).

[17] X. L. Liu, J. D. Shen, and Y. M. Xuan, Nanoscale Microscale Thermophys. Eng. 23, 188, (2019).

[18] Y. Zhang, C. L. Zhou, L. Qu, and H. L. Yi, Appl. Phys. Lett. 116, 151101 (2020).

[19] C. L. Zhou, S. H Yang, Y. Zhang, and H. L. Yi, Nanoscale Microscale Thermophys. Eng. 24, 168 (2020).

[20] B. Zhao, B. Guizal, Z. M. Zhang, S. Fan, and M. Antezza, Phys. Rev. B 95, 245437 (2017).

[21] R. Messina, P. Ben-Abdallah, B. Guizal, and M. Antezza, Phys. Rev. B 96, 045402 (2017).

[22] M. J. He, H. Qi, Y. T. Ren, Y. J. Zhao, and M. Antezza, Opt. Lett. 45, 5148 (2020).

[23] K. Z. Shi, F. L. Bao, and S. L. He, ACS Photonics 4, 971 (2017).

[24] W. Du, J. Yang, S. Zhang, N. Iqbal, Y. D. Dang, J. B. Xu, and Y. G. Ma, Nano Energy 78, 105264 (2020).

[25] K. Z. Shi, Y. C. Sun, Z. Y. Chen, N. He, F. L. Bao, J. Evans, and S. L. He, Nano. Lett. 19, 8082 (2019).

[26] X. H. Wu and R. Y Liu, ES. Energy Environ. 10, 66 (2020).

[27] J. E. Pérez-Rodríguez, G. Pirruccio, and R. Esquivel-Sirvent, Phys. Rev. Mater. 3, 015201 (2019). 
[28] G. W. Hu, A. Krasnok, Y. Mazor, C. W. Qiu, and A. Alù, Nano. Lett. 20, 3217 (2020).

[29] G. W. Hu, Q. D. Ou, G. Y. Si, Y. J. Wu, J. Wu, Z. G. Dai, A. Krasnok, Y. Mazor, Q. Zhang, Q. L. Bao, C. W. Qiu, and A. Alù, Nature (London) 582, 209 (2020).

[30] P. Li, I. Dolado, F. J. Alfaro-Mozaz, F. Casanova, L. E. Hueso, S. Liu, J. H. Edgar, A. Y. Nikitin, S. Velez, and R. Hillenbarand, Science 359, 892 (2018).

[31] J. D. Shen, S. Guo, X. L. Liu, B. A. Liu, W. T. Wu, and H. He, Appl. Therm. Eng. 144, 403 (2018).

[32] L. X. Ge, Y. P. Cang, K. Gong, L. H. Zhou, D. Q. Yu, and Y. S. Luo, AIP Adv. 8, 085321 (2018).

[33] X. L. Liu and Z. M. Zhang, Appl. Phys. Lett. 107, 143114 (2015).

[34] M. J. He, H. Qi, Y. T. Ren, Y. J. Zhao, and M. Antezza, Int. J. Heat Mass Transf. 150, 119305 (2020).

[35] M. H. Kara, A. A. Emhemed, N. A. A. Rahim, M. R. Mahmood, and Z. Awang, Microwave characterization of graphene nano-ribbon transmission lines using an improved calibration technique, in Proceedings of Tencon IEEE Region 10 Conference, Bangkok, Thailand (IEEE, Piscataway, NJ, 2014), pp. 1-6.

[36] C. Li, X. Y. Yu, T. Lan, J. Liu, and S. C Fan, IEEE Photon. Technol. Lett. 30, 565 (2018).

[37] J. S. Gomez-Diaz, M. Tymchenko, and A. Alù, Phys. Rev. Lett. 114, 233901 (2015).

[38] D. Correas-Serrano, A. Alù, and J. S. Gomez-Diaz, Phys. Rev. B 96, 075436 (2017).

[39] J. S. Gomez-Diaz and A. Alù, ACS Photonics 3, 2211 (2016).

[40] X. Lin, Z. F. Liu, T. Stauber, G. Gómez-Santos, F. Gao, H. S. Chen, B. L. Zhang, and T. Low, Phys. Rev. Lett. 125, 077401 (2020).
[41] H. Yan, X. Li, B. Chandra, G. Tulevski, Y. Wu, M. Freitag, W. Zhu, P. Avouris, and F. Xia, Nat. Nanotechnol. 7, 330 (2012).

[42] O. Ilic, M. Jablan, J. D. Joannopoulos, I. Celanovic, H. Buljan, and M. Soljacic, Phys. Rev. B 85, 155422 (2012).

[43] J. D. Shen, X. L. Liu, and Y. M. Xuan, Phys. Rev. Appl. 10, 034029 (2018).

[44] M. J. He, H. Qi, Y. F. Wang, Y. T. Ren, W.-H. Cai, and L.-M. Ruan, Opt. Express 27, A953 (2019).

[45] X. L. Liu, J. D. Shen, and Y. M. Xuan, J. Quant. Spectrosc. Radiat. Transfer 200, 100 (2017).

[46] M. J. He, H. Qi, Y. T. Ren, Y. J. Zhao, and M. Antezza, Opt. Lett. 45, 2914 (2020).

[47] M. Antezza, H. B. Chan, B. Guizal, V. N. Marachevsky, R. Messina, and M. K. Wang, Phys. Rev. Lett. 124, 013903 (2020).

[48] M. Francoeur, Near-field radiative transfer: Thermal radiation, thermophotovoltaic power generation and optical characterization, Ph.D. dissertation, University of Kentucky, 2010.

[49] Y. Zhang, C. H. Wang, H. L. Yi, and H. P. Tan, J. Quantum Spectrosc. Radiat. Transfer 221, 138 (2018).

[50] J. S. Gomez-Diaz, M. Tymchenko, and A. Alù, Opt. Mater. Express 5, 2313 (2015).

[51] M. Nakayama, J. Phys. Soc. Jpn. 36, 393 (1974).

[52] K. W. Chiu and J. J. Quinn, Phys. Rev. B 9, 4724 (1974).

[53] O. V. Kotov and Y. E. Lozovik, Phys. Rev. B 100, 165424 (2019).

[54] T. Zhan, X. Shi, Y. Dai, X. Liu, and J. Zi, J. Phys.: Condens. Matter 25, 215301 (2013).

[55] P. Yeh, J. Opt. Soc. Am. 69, 742 (1979). 